\title{
Transgender vaginoplasty: techniques and outcomes
}

\author{
Joseph J. Pariser ${ }^{1}$, Nicholas Kim ${ }^{2}$ \\ ${ }^{1}$ Department of Urology, ${ }^{2}$ Division of Plastic Surgery, University of Minnesota, Minneapolis, MN, USA \\ Contributions: (I) Conception and design: All authors; (II) Administrative support: All authors; (III) Provision of study material or patients: All authors; \\ (IV) Collection and assembly of data: All authors; (V) Data analysis and interpretation: All authors; (VI) Manuscript writing: All authors; (VII) Final \\ approval of manuscript: All authors. \\ Correspondence to: Joseph J. Pariser, MD. Department of Urology; University of Minnesota, Minneapolis, MN, USA. Email: jpariser@umn.edu.
}

\begin{abstract}
Gender affirmation surgery is paramount in the treatment of gender dysphoria for transgender individuals. For transgender women, vaginoplasty offers the opportunity for removal of masculine-appearing genitalia and replacement with a gender-congruent appearance. While numerous techniques have been described in the past, approaches have standardized considerably. Herein, we describe a technique to penile inversion vaginoplasty and focus on some of the critical steps of the procedure to try to optimize patient outcomes. We also review relevant literature regarding perioperative outcomes.
\end{abstract}

Keywords: Transgender; penile inversion; vaginoplasty; techniques; outcomes

Submitted Dec 30, 2018. Accepted for publication May 28, 2019.

doi: $10.21037 /$ tau.2019.06.03

View this article at: http://dx.doi.org/10.21037/tau.2019.06.03

\section{Preoperative considerations}

When considering vaginoplasty, patients and surgeons should adhere to WPATH guidelines for gender affirmation surgery. These include (I) persistent, well-documented gender dysphoria, (II) capacity of informed consent, (III) age of majority, (IV) well-controlled medical and mental health concerns, (V) 12 months of continuous hormones unless contraindicated, (VI) 12 months of continuously living in the gender role congruent with their gender identity, and (VII) two referrals from qualified mental health professionals. Surgery should be delayed if any of these requirements are not met.

Hair removal is critical for gender affirmation surgery. In phalloplasty, any hair-bearing skin used for the neourethra can lead to chronic infection and obstruction. In vaginoplasty, failure to perform preoperative or intraoperative hair removal can lead to inaccessible hair deep within the vagina. This can result in a hairball, which can be a nidus for debris and infection. Therefore, we generally recommend pre-vaginoplasty removal of all penile shaft hair and scrotal hair when scrotal skin use is planned. Any skin that will remain externalized does not require preoperative hair removal. Options for hair removal include electrolysis or laser hair removal. Electrolysis is an older technique involving epilation of individual hair follicles and often requires hours of treatment over months. A newer technique of laser hair removal more rapidly treats a patch of follicles by selectively targeting melanin in the hair shaft (1). There are some benefits to each approach. Hair removal tends to be more efficacious for dark hair and light skin. Patients with light hair may have inadequate clearance from laser techniques, and electrolysis would be necessary. However, a systematic review by Haedersdal and Wulf favored the efficacy of laser hair removal compared to electrolysis when feasible (2).

Venous thromboembolism (VTE) is a risk during the perioperative period of vaginoplasty. The case can be associated with a prolonged operative time in lithotomy position, especially early in the learning curve. Patients are usually on chronic estrogen supplementation. Estrogen has been associated with VTE through numerous mechanisms (3). Thus, the perioperative management of hormones is an important topic.

There is considerable variation in practice patterns related to perioperative estrogen. For example, some surgeons have patients completely hold estrogen for up to one month prior to surgery while others continue estrogen 
(often with a lower dose) throughout the perioperative period. Prospective controlled studies have not been performed and therefore, a review of some larger case series is warranted. In a study by Gaither et al., 330 transgender women underwent penile inversion vaginoplasty while on estrogen (4). Dosage was lowered to a standard $2 \mathrm{mg}$ two weeks prior to surgery. They reported no VTEs in their series, though they did not examine different methods of VTE prophylaxis. Another large series by Goddard et al. reported on the outcome of 233 patients who underwent penile inversion vaginoplasty (5). Three patients $(1.3 \%)$ experienced VTE. Notably, their protocol included cessation of feminizing hormones for 6 weeks preoperatively. Numerous other studies have been published with no patients experiencing a VTE (6).

Other modifiable factors worth mentioning are the use of chemical thromboprophylaxis and ambulation. Some surgeons utilize thromboprophylaxis liberally while others argue that it can contribute to hematoma formation. Some surgeons favor bedrest for the immediate postoperative period to improve wound healing and graft take. Others would argue that prolonged periods of bedrest contribute to VTE and pulmonary complications.

In our practice, we generally stop perioperative feminizing hormones 3-4 weeks preoperatively. We give one preoperative prophylactic dose of subcutaneous heparin and recommend early ambulation on postoperative day 1 . We utilize sequential compression devices throughout the procedure and postoperative course.

\section{Orchiectomy}

Orchiectomy represents a component of "lower" or "bottom" surgery for transgender women. It can be performed prior to vaginoplasty as a separate procedure or in conjunction with vaginoplasty. As a relatively straightforward procedure, some considerations of timing will be discussed below.

Advantages of separating orchiectomy from the rest of vaginoplasty include: patient preference (sometimes), simplification of hormone management prior to vaginoplasty, and decreased procedure time from the longer vaginoplasty procedure. Disadvantages include the need for an additional procedure and theoretical risks of increased scarring from scrotal dissection prior to vaginoplasty. In our experience, there is no noticeable difference in dissection during vaginoplasty in patients who underwent staged orchiectomy compared to those who did not.
Some patients prefer to separate the hormonal changes from the rest of vaginoplasty. For example, with the decrease in testosterone production, adjuvant hormonallyactive medications, such as spironolactone and finasteride can be stopped. Also, psychologically, some patients may simply prefer to undergo orchiectomy as a separate step to gender affirmation.

If performed as a separate procedure, orchiectomy should be performed through a midline scrotal incision to minimize incisions (e.g., subinguinal) that may impair the blood supply of the future vaginoplasty flap. Dissection continues through dartos and each hemiscrotum is entered. The cremasteric layers are transected to reach the tunica vaginalis. Dissection continues to the external inguinal ring. It is imperative to continue dissection to the ring as remnant stumps of the cord can be troubling, especially in thin patients. Once the cord is isolated at this level, it is divided in half and doubly tied. We prefer suture ligatures to ensure no slippage of suture. The cord is then transected as high as possible, and the testicle is passed off the field. The procedure is repeated on the contralateral side.

We routinely send the testicular specimen for pathology to rule out malignancy. A recent study of transgender women undergoing orchiectomy reported that despite hormonal effects, $21 \%$ of specimens demonstrated some microscopic evidence of spermatogenesis, with $4 \%$ of individuals having normal spermatogenesis. No malignant or premalignant changes were identified in 135 patients (7).

\section{Penile disassembly}

If no orchiectomy is performed, then deconstructing the penis into its parts is the first step of the operation. A circumcising incision is made. It is important to leave enough distal mucosal collar skin attached to the glans as this will allow a clitoral hood to be fashioned later. The incision should be deep enough to separate Dartos' fascia. Dissection continues between Dartos and Buck's fascia in a relatively avascular plane to the base of the penis. Once this is complete, the remainder of the penile structures can be passed into the scrotal incision, leaving just a cylinder of penile shaft skin remaining. Care should be taken to not dissect too superficially on the penile skin as it can risk its blood supply.

\section{Clitoroplasty and dorsal nerve dissection}

With the penile skin separated, the clitoroplasty is then 


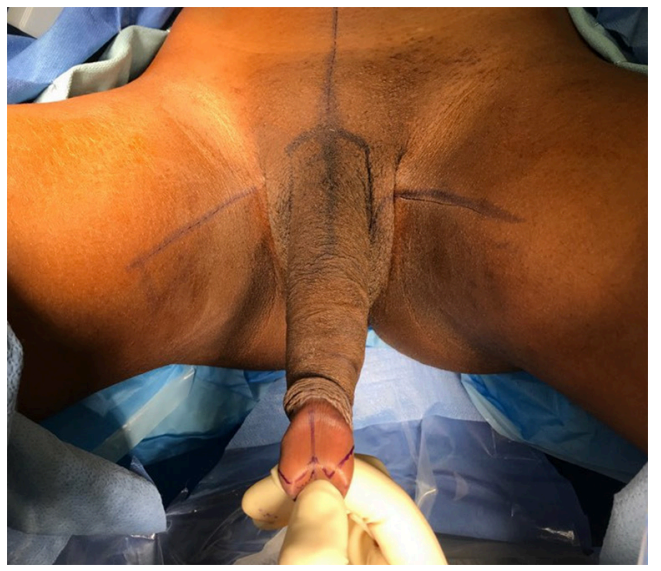

Figure 1 Preoperative vaginoplasty with " $W$ " clitoroplasty markings.

performed. A "W" shaped marking is made on the dorsal aspect of the glans (Figure 1). Surgeons should find some balance between making a small clitoris which is hidden and inaccessible and a large clitoris which resembles a glans if it is too large or its final position too prominent. An incision is made separating the $W$ shaped dorsal glans from the rest of the glans and urethra. An incision is made over the mucosal collar ventrally, and the distal collar is left attached to the future clitoris. Dissection continues down to the tips of the corporal bodies, which are located in the glans. Next, lateral incisions are made along the length of the corporal bodies. These should be sufficiently lateral to ensure that the dorsal nerves of the penis are left medially. Next, the neurovascular bundle is dissected from the remaining corporal tissue which will be discarded. There is some variability regarding the exact plane to choose. Some surgeons prefer to dissect right on the outside of the dorsal aspect of the tunica albuginea. This can be somewhat tedious and risks injury to the dorsal nerves. Alternatively, we dissect on the underside of the dorsal tunica albuginea. This is a rapid dissection in the corpus cavernosa itself. Dissection continues literally along the pre-incised markings and proximally to the level of the pubic symphysis. Adequate proximal dissection is necessary or patients can experience an erection of their corporal stump, which is undesired. We oversaw the proximal stumps with absorbable sutures. Of note, the ventral aspect of the corpora cavernosa stump is a free edge while the dorsal aspect is a sheet (not an edge) of tunica albuginea as it was not transected, Thus, the ventral edge of tunica is sewn to the sheet of dorsal tunica albuginea with care not to completely traverse the tunica and potentially ligate the nerve on the dorsal side of the dorsal tunica.

Clitoroplasty then continues by rolling the "W" shaped reduced glans at the midline ventrally. The pedicle is then accordioned and the clitoris is suture suspended from the pubic symphysis at the midline, placed above the corporal stumps. The folded pedicle is then sutured down to the suprapubic abdominal fascia without injuring the neurovascular bundle by taking longitudinal bites with the needle. The dorsal collar of the penile skin is then folded over the clitoris to reconstruct the clitoral hood. The lateral wings are then brought together at the midline underneath the clitoris and sutured together to complete the clitoroplasty.

\section{Urethroplasty}

Urethral dissection can begin anywhere distal to the bulbar urethra. We generally start in the pendulous urethra. Circumferential isolation of the urethra allows for mobilization away from the dorsally located corporal bodies. This is deep to Buck's fascia. As dissection continues to the distal bulbar urethra, the bulbospongiosus muscle is encountered. Management of this muscle varies. We prefer complete medial and lateral release from the corpus spongiosum and surrounding structures. The muscle is left on its attachments posteriorly to allow for a wellvascularized flap in the rare event of a rectal injury.

Circumferential urethral dissection is generally continued proximally to the distal bulbar urethra. The urethra is transected roughly $2-3 \mathrm{~cm}$ distal to the turn of the bulbar urethra. The urethra is then spatulated ventrally for $2-3 \mathrm{~cm}$. The dorsal plate of this spatulated urethra is then flipped up and sutured to the neoclitoris and clitoral hood. This creates a vulvar vestibule composed of urethral mucosa along with a well-spatulated urethra. It is important to ensure spatulation is taken proximally enough that the urethra is not pointed upward when sitting to void.

Later, a "second hole" or anteriorly located opening in the skin flap (above the "hole" of the inverted penile skin) is made to allow for exposure of the clitoris and the urethra. The urethral mucosa is matured to the skin edge ventrally and laterally to this opening using absorbable suture. Prior to maturation, some surgeons advocate oversewing the edge of the corpus spongiosum to decrease postoperative 
bleeding. A catheter is left in place postoperatively.

\section{Perineal flap augmentation of vaginal introitus}

Just inferior to the perineal body, a $2 \mathrm{~cm} \times 2 \mathrm{~cm}$ posteriorly based triangular shaped flap is made, which will later create a posterior fourchette and connected to the midline inferior edge of the penile skin tube. We generally place some sutures to prevent this area from prolapsing in the postoperative period and creating an undesired fullness in the posterior fourchette.

\section{Creation of vaginal cavity}

The most daunting step of a vaginoplasty is the creation of the neovaginal canal given the risk of rectal and urethral injury. Preoperatively, all patients should be counseled regarding the possibility of "limited depth" vaginoplasty, which entails dissection to the membranous urethra. Patients who are not interested in penetrative forms of sex, higher perioperative risk or are not willing to commit to lifelong douching and dilating should be considered ideal candidates for limited depth vaginoplasty. This leaves the patient with an externally feminine genitalia with a very short vaginal canal of just a few centimeters inadequate for penetrative intercourse. However, it mitigates the risk of rectal and urethral injury, obviates the need for supplemental grafts or flaps for additional vaginal length (and thus hair removal needs), and shortens the operative time.

Standard perineal approach to neovaginal canal creation involves dissection of the bulbospongiosus muscle. We generally prefer to detach this muscle laterally and leave it attached posteriorly to allow it to be used as an interposition flap if a rectal injury is made later. Dissection continues to the central tendon, which is divided transversely. This allows mobility to the bulbar urethra. We prefer to place a lubricated rectal condom drape to allow sterile finger guidance to perirectal dissection.

Establishing the correct plane at this point is critical. We prefer sharp dissection using Metzenbaum scissors while finding a plane just anterior to Denonvilliers' fascia with intermittent reassessment of depth of tissue to the finger in the rectum and urethral catheter palpation. Some authors describe using additional retractors in the urethra, such as a Lowsley (8), but we have found such instruments can alter the usual anatomic configuration. It is very easy at this point to mistakenly traverse the prostatic capsule and dissect in an intraprostatic fashion. If this occurs, the dissection will become difficult and the catheter will be palpated very closely. Full dissection of a canal will not be possible until the surgeon reestablishes the plane external to the prostatic capsule. Urethral injury is possible if dissection continues in an intraprostatic plane, though usually a two-layer closure leads to no significant consequence. Denonvilliers' fascia (or the rectoprostatic fascia) should be left intact during this dissection. Inadvertent traversing of Denonvilliers' will lead to dissection in mesorectal fat, which should be obvious to the surgeon. Longitudinal rectal fibers may also be seen, which should prompt the surgeon to re-establish the correct plane. Once the plane on the prostatic capsule is identified, blunt dissection often promptly leads the surgeon to the peritoneal reflection, ending the dissection. At the end of dissection, a purple Soul Source \#7 vaginal dilator (Soul Source, North Hollywood, CA) should be accommodated in the cavity with minimal effort. This dilator measures $5-1 / 2$ inches in length and 1-7/16 inches in diameter.

\section{Augmentation of the neovagina}

Most patients, especially those who have undergone previous circumcision, require some graft or flap to augment the neovagina in order to line the canal. Various approaches have been taken. The most common method is to utilize a skin graft from the scrotal skin, which would usually be discarded anyways. This can be done in a split thickness or full thickness approach. Preoperative hair removal is critical to preventing postoperative hair accumulation at the apex of the vagina. Two triangle shaped scrotal skin wedges are excised once the neovaginal construct is positioned appropriately. On the back table, the graft is aggressively thinned and defatted. Once ready, the edges of the grafts are then approximated over a \#7 dilator to form a "cap". The proximal edge of the graft is then sutured to the distal aspect of the penile shaft skin. We favor some relaxing incisions on the penile shaft skin laterally to limit cicatricial stenosis from a symmetric, circular anastomosis.

An exciting alternative to scrotal skin graft is a peritoneal flap. Peritoneal vaginoplasty has gained popularity recently in transgender cases given its lack of donor site morbidity, hairless nature and availability though the use of peritoneum was initially described in 1969 by Davydov (9). Laparoscopic and robotic approaches have decreased the morbidity of peritoneal flap harvest. Additionally, these transabdominal approaches can aid in the neovaginal canal dissection. Peritoneal flaps are usually sewn to the inverted penile skin flap, obviating the need for scrotal 


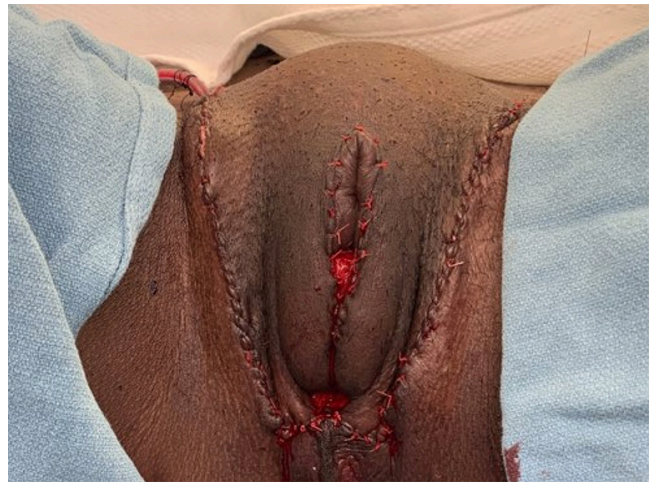

Figure 2 Immediate postoperative vaginoplasty.

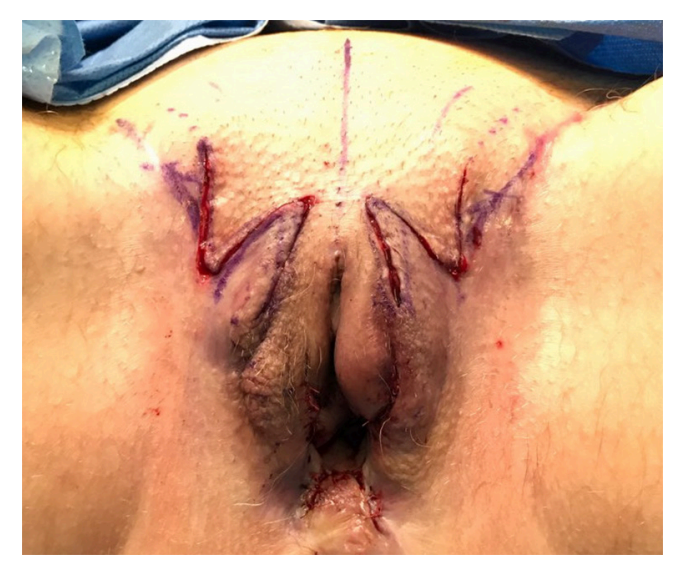

Figure 3 Intraoperative second stage labiaplasty.

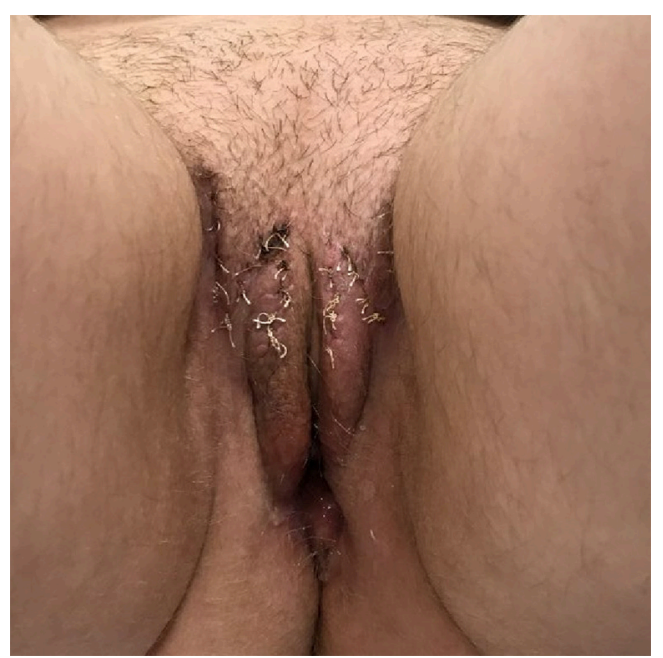

Figure 4 Early postoperative healing after second stage labiaplasty (same patient). skin grafts. A series of primary and revision vaginoplasties using peritoneal flaps via a robotic approach was recently published (10).

Another alternative for tissue is an intestinal substitution. For example, a sigmoid neovagina has been described given its proximity to the pelvis $(11,12)$. Other positives include its hairless nature and self-lubrication. However, intestinal neovagina has fallen out of favor for transgender women given the large amount of mucous production, morbidity associated with bowel harvest and chronic foul odor.

\section{Labiaplasty}

During the initial surgery, triangular excision of excess scrotal tissue is performed bilaterally. This leaves a "U" shaped incision to close (Figure 2). This leaves a feminine vagina. However, some patients desire a more defined labia majora. Also, some patients have issues with wound dehiscence.

The labia minora is fashioned out of penile shaft dorsal collar skin. The labia majora is fashioned out of remnant scrotal tissue after its excess is removed through a lateral incision that is at least $1 \mathrm{~cm}$ away from the groin crease. The blood supply to the penile skin tube being anteriorly based, we do not recommend placing incisions along the pubic region at the time of vaginoplasty. As a result, the reconstructed labia majora will maintain a laterally diverging appearance at their anterior aspect. If needed, this can be alleviated with a second stage feminizing labiaplasty to bring the anterior limbs of the labia majora together through the use of transposition flaps (Figures 3,4). This produces a lenticular shape to the external vulva, better defines the labia minora, and produces a deeper clitoral hood.

It is noted that superficial wound dehiscence along the posterior-most edge of the labia majora is a common complication that occurs in almost every case. This adds to the importance of meticulous layered closure and careful postoperative hygiene around that region. In addition, we utilize channeled JP drains that enter at the anterior corners of the labia majora to decrease fluid collections that may place pressure on the closure.

\section{Postoperative considerations}

Our postoperative protocol includes a hospital admission. We typically leave sutured-in vaginal packing in place 
for 5 days postoperatively. We also leave two closed suction drains and a urethral catheter in place. On the 5 th postoperative day, the packing is removed. If no other abnormalities are noted, then the urethral catheter and drains are removed, and the patient is discharged after successful voiding. Patients begin dilating on their first clinic visit at 7 to 10 days postop. Our protocol includes 3 months of 3 times daily 15 -minute dilations, followed by 6 months of 2 times daily dilations. Eventually, patients are recommended to perform lifelong dilations every one to two days. Our patients are reminded to use a clean dilator each time without touching the anus and to douche the vaginal cavity after each dilation.

\section{Outcomes}

Outcomes of penile inversion vaginoplasty are generally in the form of large case series from high volume institutions. The largest series $(n=475)$ from Amsterdam recently reported on their experience (13). They reported a standard technique, similar to what is described in this manuscript. Of note, $85 \%$ required the use of additional skin grafts. Median follow-up was 7.8 years. They reported a $2.3 \%$ rate of rectal injury with a $0.6 \%$ overall rate of rectoneovaginal fistula. They also reported a $1.1 \%$ rate of intraoperative urethral injury and a $1.7 \%$ rate of postoperative urethroneovaginal fistula. This is likely due to some injuries being identified and repaired intraoperatively. Minor necrosis occurred in $24.6 \%$ of patients with only $0.6 \%$ of patients classified as "major" necrosis requiring reoperation under general anesthesia. The presence of diabetes was associated with local infection or wound breakdown.

These outcomes are consistent with other published series. Rectal injury, which can be devastating, has been reported in $0.4-4.5 \%$ of patients in other series $(4,5,14-20)$. Urethral injury is less frequently reported, though a few series have reported rates of $1.1 \%$ to $3.6 \%(13,18)$. Urethroneovaginal fistula can be a long-term consequence of a urethral injury and can lead to incontinence. Fistulas below the external sphincter are often less consequential as patients are still continent.

Long-term, stenosis of the neovagina is often related to discontinuation of dilation. Regardless, it has been reported in the $2-12 \%$ range. This may be underreported as these events can occur long after initial vaginoplasty if patients stop performing dilation.

Meatal stenosis can be seen postoperatively. There is some variation in the handling of the urethra during vaginoplasty. We prefer a long ventral spatulation, thus creating a wide-open anastomosis and a mucosal vestibule. However, others perform an "end" urethrostomy, thus creating a small mucosal eversion, which we feel is more likely to lead to stenosis. Various series have reported a meatal stenosis rate of $1-39 \%$. However, studies on the higher end of meatal stenosis rate favored this "end urethrostomy" approach $(5,18)$. In follow-up, meatal stenosis can be readily corrected with a ventral urethrotomy with mucosal eversion or a ventral Y-V plasty of the meatus with high success.

\section{Conclusions}

Vaginoplasty is a relatively safe procedure in experienced hands. The major source of high-grade complications results from the neovaginal canal dissection, which can be treacherous given the proximity to the urethra and rectal structures. A high index of suspicion is needed to identify intraoperative injuries as immediate repair can lower the chance of fistula. Wound dehiscence is relatively common, though it is usually managed nonoperatively. In our experience, the wound dehiscence occurs most frequently at the inferior most aspect of the lateral labial closures. We suspect this area is under the most tension during closure. Long-term complications, such as neovaginal stenosis usually result from cessation of dilation. Standardized patient centered outcomes measures are greatly needed for vaginoplasty to compare results and standardize techniques between centers.

\section{Acknowledgments}

None.

\section{Footnote}

Conflicts of Interest: The authors have no conflicts of interest to declare.

\section{References}

1. Zhang WR, Garrett GL, Arron ST, et al. Laser hair removal for genital gender affirming surgery. Transl Androl Urol 2016;5:381-7.

2. Haedersdal M, Wulf HC. Evidence-based review of hair removal using lasers and light sources. J Eur Acad Dermatol Venereol 2006;20:9-20. 
3. Tchaikovski SN, Rosing J. Mechanisms of estrogeninduced venous thromboembolism. Thromb Res 2010;126:5-11.

4. Gaither TW, Awad MA, Osterberg EC, et al. Postoperative Complications following Primary Penile Inversion Vaginoplasty among 330 Male-to-Female Transgender Patients. J Urol 2018;199:760-5.

5. Goddard JC, Vickery RM, Qureshi A, et al. Feminizing genitoplasty in adult transsexuals: early and long-term surgical results. BJU Int 2007;100:607-13.

6. Horbach SE, Bouman MB, Smit JM, Get al. Outcome of Vaginoplasty in Male-to-Female Transgenders: A Systematic Review of Surgical Techniques. J Sex Med 2015;12:1499-512.

7. Kent MA, Winoker JS, Grotas AB. Effects of Feminizing Hormones on Sperm Production and Malignant Changes: Microscopic Examination of Post Orchiectomy Specimens in Transwomen. Urology 2018;121:93-6.

8. Kocjancic E, Vigneswaran H, Sofer L, et al. Gender confirmation vaginoplasty: The Chicago technique. Eur Urol Suppl 2018;17:e1991.

9. Davydov SN. Colpopoeisis from the peritoneum of the uterorectal space. Akush Ginekol (Mosk) 1969;45:55-7.

10. Jacoby A, Maliha S, Granieri MA, et al. Robotic Davydov Peritoneal Flap Vaginoplasty for Augmentation of Vaginal Depth in Feminizing Vaginoplasty. J Urol 2019;201:1171-6.

11. Salgado CJ, Nugent A, Kuhn J, et al. Primary Sigmoid Vaginoplasty in Transwomen: Technique and Outcomes. BioMed Res Int 2018;2018:4907208.

12. Bouman MB, van der Sluis WB, Buncamper ME, et

Cite this article as: Pariser JJ, Kim N. Transgender vaginoplasty: techniques and outcomes. Transl Androl Urol 2019;8(3):241-247. doi: 10.21037/tau.2019.06.03 al. Primary Total Laparoscopic Sigmoid Vaginoplasty in Transgender Women with Penoscrotal Hypoplasia: A Prospective Cohort Study of Surgical Outcomes and Follow-Up of 42 Patients. Plast Reconstr Surg 2016;138:614e-23e.

13. Buncamper ME, van der Sluis WB, van der Pas RSD, et al. Surgical Outcome after Penile Inversion Vaginoplasty: A Retrospective Study of 475 Transgender Women. Plast Reconstr Surg 2016;138:999-1007.

14. Perovic SV, Stanojevic DS, Djordjevic ML. Vaginoplasty in male transsexuals using penile skin and a urethral flap. BJU Int 2000;86:843-50.

15. Krege S, Bex A, Lümmen G, et al. Male-to-female transsexualism: a technique, results and long-term followup in 66 patients. BJU Int 2001;88:396-402.

16. Wagner S, Greco F, Hoda MR, et al. Male-to-female transsexualism: technique, results and 3-year follow-up in 50 patients. Urol Int 2010;84:330-3.

17. Reed HM. Aesthetic and functional male to female genital and perineal surgery: feminizing vaginoplasty. Semin Plast Surg 2011;25:163-74.

18. Rossi Neto R, Hintz F, Krege S, et al. Gender reassignment surgery--a 13 year review of surgical outcomes. Int Braz J Urol 2012;38:97-107.

19. Raigosa M, Avvedimento S, Yoon TS, et al. Male-toFemale Genital Reassignment Surgery: A Retrospective Review of Surgical Technique and Complications in 60 Patients. J Sex Med 2015;12:1837-45.

20. Wangjiraniran B, Selvaggi G, Chokrungvaranont $P$, et al. Male-to-female vaginoplasty: Preecha's surgical technique. J Plast Surg Hand Surg 2015;49:153-9. 